JoÃo Paulo SARTORI ${ }^{1}$

José ANTÔNIO MORAEs Martins ${ }^{1}$

Rodrigo de AQUINO CASTRO ${ }^{1}$

Marair Gracio Ferreira Sartorl ${ }^{2}$

Manoel joão Batista Castello Girão ${ }^{3}$

Artigos originais

Palavras-chave

Incontinência urinária por estresse/cirurgia

Slings suburetrais

Procedimentos cirúrgicos

urológicos/métodos

Procedimentos cirúrgicos minimamente invasivos

Keywords

Urinary incontinence, stress/surgery Suburethral slings Urologic surgical procedures/methods Surgical procedures, minimally invasive

\section{Sling de aponeurose e com faixa sintética sem tensão para o tratamento cirúrgico da incontinência urinária de esforço feminina}

\author{
Pubovaginal sling and tension-free vaginal tape for surgical treatment \\ of stress urinary incontinence in women
}

\title{
Resumo
}

OBJETIVO: comparar as cirurgias de sling de aponeurose e TVT (do inglês tension-free vaginal tape) para correção da incontinência urinária de esforço (IUE) quanto às taxas de cura subjetiva e objetiva, à mobilidade do colo vesical ao ultra-som, à variação do teste do absorvente, às alterações urodinâmicas e à incidência de complicações. MÉTODOS: foram selecionadas 80 pacientes com IUE. Destas, 61 foram operadas pela técnica de TVT e 19 por sling de aponeurose do reto abdominal. As médias de idade, índice de massa corpórea e paridade foram 50, 1 anos, $29,7 \mathrm{~kg} / \mathrm{m}^{2}$ e 4,5 partos (mediana=3) para as pacientes com sling de aponeurose e de 51,7 anos, 28, $1 \mathrm{~kg} / \mathrm{m}^{2} \mathrm{e}$ 4, 1 partos (mediana=3) para aquelas com TVT. Todas se submeteram a anamnese, exame físico, ultra-sonografia do colo vesical, teste do absorvente e estudo urodinâmico no pré-operatório e após, pelo menos, seis meses depois da cirurgia. Após 15 ou 19 meses e depois de cerca de quatro a cinco anos, foram novamente entrevistadas quanto aos resultados da cirurgia. RESULTADOS: quanto à avaliação subjetiva, após seis meses, julgaram-se curadas 96,7\% das mulheres com TVT e 89,5\% das com sling. Porém, após 15 a 19 meses, o Grupo TVT manteve a mesma taxa de cura subjetiva, enquanto que no Grupo Sling houve redução para 77,8\%. Houve diminuição significativa da mobilidade do colo vesical, similar em ambos os grupos, e melhora no teste do absorvente. No final do estudo urodinâmico, foram classificadas como cura objetiva 93,4\% das mulheres do Grupo TVT e 78,9\% daquelas do Grupo Sling. $\bigcirc$ tempo médio de sondagem vesical foi maior no Grupo Sling. Observou-se retenção urinária em 42, 1\% dos casos de sling e em $9,8 \%$ de TVT. As taxas de cura tardia foram $90 \%$ para TVT e 55,6\% para sling. CONCLUSÕES: a cirurgia de TVT propiciou melhor taxa de cura subjetiva após 15 ou 19 meses, porém, a taxa de cura objetiva foi igual em ambas as técnicas neste tempo. Entre as complicações detectadas, a retenção urinária no pós-operatório foi superior no Grupo Sling.

\section{Abstract}

PURPOSE: to compare sling operations of aponeurosis and tension-free vaginal tape (TVT) for the correction of stress urinary incontinence (SUI) regarding: the rates of subjective and objective healing, the mobility of the bladder neck with ultrasonography, the variation of the absorbent test, the urodynamic alterations and the incidence of complications. METHODS: eighty patients with SUI were selected. Among them, 61 underwent a TVT surgery and 19, an abdominal rectum sling operation of aponeurosis. Average age, index of body mass and parity were 50.1 years old, $29.7 \mathrm{~kg} / \mathrm{m}^{2}$ and 4.1 deliveries (median=3) for the patients with aponeurosis sling, and 51.7 years old, $28.1 \mathrm{~kg} / \mathrm{m}^{2}$ and 4. 1 deliveries (median=3) for the ones with TVT. All of them have undergone anamnesis, physical examination, bladder neck ultrasonography, absorbent test and urodynamic evaluation before and at least six months after the surgery. After 15 or 19 months and after about four or five years, they were again interviewed concerning the surgery results. RESULTS: after six months, $96.7 \%$ of the women with TVT and $89.5 \%$ of the ones with sling thought they were healed in the subjective evaluation. Nevertheless, after 15 to 19 months, the TVT Group kept the same subjective healing rate, while among the Sling Group the rate decreased to $77.8 \%$. There was a significant decrease in the mobility of the neck bladder that was similar in both groups and an improvement in the absorbent test. At the end of the urodynamic study, 93.4\% of the women from the TVT Group and 78.9\% of the ones from the Sling Group were classified as having an objective healing. The average time of bladder probing was higher in the Sling Group. Urinary retention was

Correspondência: João Paulo Sartori Rua Dr. Diogo de Faria, 1.087, ci. 1.102 CEP 04037-003 - São Paulo/SP E-mail: jsartor@@ul.com.br
Trabalho realizado no Departamento de Ginecologia da Universidade Federal de São Paulo - UNIFESP - São Paulo (SP), Brasil. ' Pós-graduandos do Departamento de Ginecologia da Universidade Federal de São Paulo - UNIFESP - São Paulo (SP), Brasil. 2 Livre-Docente, Professora Associada do Departamento de Ginecologia da Universidade Federal de São Paulo - UNIFESP - São Paulo (SP), Brasil.

3 Professor Titular do Departamento de Ginecologia da Universidade Federal de São Paulo - UNIFESP - São Paulo (SP), Brasil. 
observed in $42.1 \%$ of the sling cases and in $9.8 \%$ of the TVT's. The rates of late healing were $90 \%$ for TVT and $55.6 \%$ for sling. CONCLUSIONS: TVT surgery provided better subjective healing after 15 or 19 months, but the rate of objective healing was the same in both techniques at that time. Among the complications detected, the urinary retention was higher in the Sling Group, in the post-surgery period.

\section{Introdução}

A incontinência urinária de esforço (IUE) é conceituada como toda perda de urina pelo meato externo da uretra sincrônica ao esforço físico, como tossir ou espirrar. É classificada em IUE por hipermobilidade do colo vesical e a por decorrência de defeito esfincteriano da uretra ${ }^{1}$.

A cirurgia representa, ainda, a principal forma terapêutica da incontinência de esforço. Porém, a existência de inúmeras técnicas para a sua correção mostra que o tema não está totalmente esclarecido, pois, mesmo com adequada avaliação pré-tratamento, há recidivas, o que desafia os que se propõem a tratá-la ${ }^{2}$. A escolha da técnica deve levar em consideração o correto diagnóstico, os índices de recidiva, a integridade do sistema esfincteriano uretral, bem como a experiência e a segurança do cirurgião com cada procedimento.

A incontinência urinária por hipermobilidade pode ser tratada por procedimento cirúrgico que recoloque a uretra proximal e o colo vesical em posição retropúbica, alta e fixa, com excelentes resultados, como a colpofixação retropúbica pela técnica de Burch ${ }^{3}$. Entretanto, quando há lesão do esfíncter, a cirurgia de alça (sling) assume importante papel, pois atua também estabilizando e criando um novo suporte para a uretra. Nesta técnica, coloca-se faixa de material autólogo ou heterólogo na região suburetral, próxima à junção uretrovesical ${ }^{4,5}$. Assim, na vigência de aumento da pressão intra-abdominal, a uretra é forçada contra esta faixa, fechando-se e mantendo a continência.

A cirurgia de sling também é indicada para o tratamento das recidivas pós-operatórias da técnica de Burch e para pacientes com alto risco de falha cirúrgica, como as que têm bronquite crônica, asma, flacidez congênita dos tecidos ou que praticam atividades físicas de alto impacto, as com obesidade severa ou em uso crônico de corticóide ${ }^{6}$. Pode, contudo, ser aplicada a todos os tipos de incontinência de esforço ${ }^{5}$. É contra-indicada para pacientes com disfunções miccionais, como bexiga flácida, grande resíduo pós-miccional, bexiga hiperativa, refluxo vesicoureteral ou fístulas urogenitais 5 .

As taxas de cura ou melhora variam entre 49 e $92 \%$, mas se acompanham de alta incidência de retenção urinária prolongada e de bexiga hiperativa secundária. No entanto, proporciona alto grau de satisfação às pacientes, a despeito de eventuais desconfortos no pós-operatório ${ }^{7,9}$. Segundo Silva-Filho et al. ${ }^{10}$, em pacientes com defeito esfincteriano intrínseco, o sling pubovaginal também obtém resultados adequados, com cerca de $88 \%$ de melhora objetiva, porém, com baixas taxas de retenção urinária.
Com o intuito de tratar a IUE por cirurgia menos invasiva e com menor incidência de distúrbios miccionais no pós-operatório, desenvolveram-se os slings sintéticos. Nesse grupo, destaca-se a técnica cirúrgica denominada TVT (do inglês tension-free vaginal tape), por via retropúbica. Foi desenvolvida baseando-se na teoria integral para a IUE ${ }^{11}$, consoante a qual a perda do sistema de sustentação da vagina por meio dos ligamentos pubouretrais seria responsável pela maior parte dos distúrbios miccionais. Assim, desenvolveu-se alça de prolene, que pode ser posicionada no espaço retropúbico e enlaça a uretra média ${ }^{12}$.

Os índices de sucesso são elevados, com nítido ganho no pós-operatório imediato e tardio, tanto para casos primários quanto nas recidivas ou nos casos de defeito esfincteriano intrínseco. Promove nítida redução da urgência miccional pré-operatória, o que outras técnicas, além de não melhorarem, podem até acentuar ou desenvolver. Tem sido considerada, atualmente, como cirurgia de escolha em vários países da Europa ${ }^{13-16}$.

O TVT vem sendo comparado com outras técnicas para correção da IUE, como a cirurgia de Burch, considerada como padrão-ouro no tratamento desta afecção e que apresenta resultados avaliáveis em longo prazo ${ }^{17}$. Estudos multicêntricos, randomizados, comparando ambas as técnicas, mostraram resultados similares; porém, os benefícios do TVT foram: menor duração do procedimento, tempo de hospitalização menor e retorno mais rápido às atividades de rotina ${ }^{18-23}$. Liapis et al. ${ }^{18}$, em estudo randomizado e prospectivo, identificaram taxas de cura similares entre a cirurgia de Burch e o TVT, mas o tempo operatório, o índice de dor e o tempo necessário para o retorno às atividades normais foram menores no Grupo TVT.

Em nosso meio, muitos serviços não dispõem de faixas sintéticas para o tratamento da IUE, utilizando as faixas autólogas da aponeurose do reto abdominal. Assim, objetivamos analisar ambas as técnicas no tratamento dessa afecção, quanto aos resultados e às complicações.

\section{Métodos}

Trata-se de estudo prospectivo em que foram selecionadas 80 pacientes com IUE atendidas no Setor de Uroginecologia e Cirurgia Vaginal da Disciplina de Ginecologia Geral do Departamento de Ginecologia da Escola Paulista de Medicina da Universidade Federal de São Paulo (UNIFESP/EPM), entre 1999 e 2006.

Foram incluídas mulheres com IUE comprovada por exame físico ou por estudo urodinâmico, que optaram por 
tratamento cirúrgico. Não foram incluídas mulheres com bexiga neurogênica flácida, com infecção urinária recente, que não desejassem cirurgia como tratamento e que não concordaram em assinar o termo de consentimento livre e esclarecido. Os casos com IUE que necessitavam também de laparotomia para tratamento de doenças pélvicas, como leiomioma ou doenças do ovário, também não foram incluídos. A presença de contrações não inibidas do detrusor não foi considerada como critério de não inclusão, e foi encontrada em apenas uma paciente no pré-operatório do Grupo TVT.

As pacientes foram avaliadas com exame de urina tipo I, urocultura, ultra-sonografia do colo vesical e estudo urodinâmico. Aquelas com infecção urinária foram tratadas e, somente após negativação da urocultura, prosseguiram com a propedêutica subsidiária.

O estudo urodinâmico foi realizado para o diagnóstico da incontinência urinária. As definições, técnicas e unidades foram padronizadas segundo a Sociedade Internacional de Continência ${ }^{1}$. Assim, considerou-se a perda de urina durante a cistometria como indicador de IUE. Pesquisou-se a pressão abdominal de perda (Valsalva leak point pressure) durante manobra de Valsalva realizada com volume vesical de $200 \mathrm{~mL}$. Quando essa pressão fosse inferior a $60 \mathrm{cmH}_{2} \mathrm{O}$, considerava-se como defeito esfincteriano intrínseco.

No dia em que foram internadas para cirurgia, foi realizado o teste do absorvente simplificado de 20 minutos, para quantificar a perda urinária ${ }^{24}$.

As pacientes com indicação cirúrgica eram encaminhadas para a internação no Hospital São Paulo. Segundo a rotina do Setor, os casos eram discutidos em reunião multidisciplinar, em que era indicado o tipo de cirurgia. As pacientes receberam a indicação de cirurgia de alça e foram operadas por sling de aponeurose ou TVT, de acordo com a disponibilidade da faixa sintética no Hospital São Paulo.

Os grupos foram homogêneos em relação a idade, índice de massa corpórea (IMC), número de gestações, número de partos normais, de partos a fórcipe, de partos cesárea, número de abortamentos e tempo de perda de urina (Tabela 1). Eram da raça branca $86,9 \%$ das pacientes operadas com TVT e 89,4\% daquelas com cirurgia de sling de aponeurose $(\mathrm{p}=0,7)$.

A hipermobilidade do colo vesical foi observada por meio de ultra-sonografia, quando a descida do colo vesical em relação à borda inferior da sínfise púbica foi superior a $10 \mathrm{~mm}$. Considerou-se defeito esfincteriano intrínseco ou incontinência urinária tipo III quanto a pressão abdominal na manobra de Valsalva, realizada com $200 \mathrm{~mL}$ de volume vesical, era igual ou menor que $60 \mathrm{cmH}_{2} \mathrm{O}^{1}$.

Foram operadas pela técnica de sling de aponeurose 19 pacientes e, pela técnica de TVT retropúbico, outras 61. Apresentavam hipermobilidade do colo vesical 18 $(94,7 \%)$ das pacientes com sling e $53(86,9 \%)$ daquelas com TVT. O defeito esfincteriano intrínseco foi identificado em dez $(52,6 \%)$ das mulheres com sling e em $16(26,2 \%)$ das com TVT.

Os procedimentos cirúrgicos foram realizados por médicos pós-graduandos do Setor de Uroginecologia e Cirurgia Vaginal, sob supervisão do corpo docente. O seguimento pós-operatório foi realizado sempre pelo mesmo pesquisador.

A cirurgia com TVT retropúbico foi realizada seguindo a técnica descrita por Petros ${ }^{25}$. Para a cirurgia de sling de aponeurose do músculo reto anterior do abdome, utilizou-se a técnica descrita por McGuire e Lytton ${ }^{26}$, com retalho de faixa da aponeurose com $12 \mathrm{~cm}$ de comprimento e $1 \mathrm{~cm}$ de largura.

No Grupo Sling, todas as cirurgias foram realizadas com bloqueio espinhal. No Grupo TVT, seis $(9,8 \%)$ pacientes foram operadas com raquianestesia e as demais 55 (90,2\%), com anestesia local e sedação. Optou-se pelo bloqueio intradural em dois casos na fase de treinamento da equipe, em duas mulheres com obesidade que impediu a anestesia local, em um caso que necessitou de perineotomia prévia e em outro no qual foi feita uretrólise antes do TVT. Em 20 pacientes, depois de terminado o TVT com anestesia local, procedeu-se ao bloqueio espinhal para realização de outra cirurgia associada, sendo colporrafia posterior e perineorrafia em 19 casos e curetagem uterina em uma paciente. Das pacientes que se submeteram a sling de aponeurose, três tinham prolapso uterino, realizando-se histerectomia em duas delas e, em uma, cirurgia de Manchester.

No pós-operatório, as pacientes foram examinadas entre o sexto e o oitavo dia para retirada dos pontos e/ou retirada da sonda vesical. Em seguida, foram avaliadas mensalmente, por pelo menos seis meses. $\mathrm{O}$ seguimento médio foi de 19 meses após TVT e de 15 meses após sling.

Tabela 1 - Pacientes com incontinência urinária de esforço tratadas pelas cirurgias de TVT ou sling de aponeurose, quanto à idade, raça, índice de massa corpórea (IMC), número de gestações (G), de partos normais (PN), de partos fórcipe (PF), de partos cesárea $(\mathrm{PC})$, de abortos (A) e tempo de perda de urina (TP) em anos

\begin{tabular}{|c|c|c|c|c|c|c|}
\hline & \multicolumn{3}{|c|}{ TVT } & \multicolumn{3}{|c|}{ Sling } \\
\hline & Variação & Média & $d p$ & Variação & Média & $d p$ \\
\hline Idade (anos) & $34-79$ & 51,7 & 11,5 & $24-71$ & 50,1 & 13,5 \\
\hline IMC $\left(\mathrm{kg} / \mathrm{m}^{2}\right)$ & 19-39 & 28,1 & 4,9 & $21-40$ & 29,7 & 5,0 \\
\hline$G\left(n^{0}\right)$ & 0.15 & 4,8 & 3,2 & $1-11$ & 5,2 & 3,5 \\
\hline PN $\left(n^{\circ}\right)$ & $0-13$ & 3,5 & 3,1 & $0-9$ & 3,9 & 3,1 \\
\hline $\operatorname{PF}\left(n^{0}\right)$ & $0-2$ & 0,1 & 0,4 & $0-1$ & 0,1 & 0,3 \\
\hline $\mathrm{PC}\left(\mathrm{n}^{\circ}\right)$ & $0-2$ & 0,5 & 0,8 & $0-3$ & 0,4 & 0,8 \\
\hline$A\left(n^{0}\right)$ & $0-6$ & 0,7 & 1,2 & $0-2$ & 0,7 & 1,0 \\
\hline TP (anos) & $1-30$ & 7,3 & 5,7 & $1-30$ & 7,0 & 7,2 \\
\hline \multicolumn{3}{|c|}{ Idade: TVT=Sling $(p=0,598)$} & \multicolumn{4}{|c|}{ IMC: TVT=Sling $(p=0,208)$} \\
\hline \multicolumn{3}{|c|}{ G: TVT=Sling $(p=0,648)$} & \multicolumn{4}{|c|}{ PN: TVT=Sling $(p=0,570)$} \\
\hline \multicolumn{3}{|c|}{ PF: TVT=Sling $(p=0,776)$} & \multicolumn{4}{|c|}{$P C:$ TVT=Sling $(p=0,896)$} \\
\hline \multicolumn{3}{|c|}{ A: TVT=Sling ( $\mathrm{p}=0,902)$} & \multicolumn{4}{|c|}{ TP: TVT=Sling $(p=0,809)$} \\
\hline
\end{tabular}


Após pelo menos seis meses, as pacientes que consentiram fizeram novamente o teste do absorvente, a ultra-sonografia do colo vesical e o estudo urodinâmico.

Todas as pacientes com sling permaneceram com sondagem vesical durante sete dias, recebendo antiinflamatório, antibiótico, analgésico e anti-séptico das vias urinárias. Se a paciente não conseguisse urinar após a retirada da sonda, recomendavam-se novas sondagens por pelo menos sete dias e assim sucessivamente, podendo-se associar ansiolíticos (benzodiazepínicos), agentes colinérgicos e bloqueadores alfa-adrenérgicos. Considerou-se como retenção urinária quando o tempo de sondagem foi superior a sete dias.

No Grupo TVT, a sonda vesical permaneceu por 24 horas nos casos em que se realizou bloqueio espinhal. Nos casos com anestesia local, a sonda foi retirada na sala cirúrgica. Considerou-se como retenção urinária quando houve necessidade de sondagem vesical além desses prazos.

A cada consulta e após cerca de seis meses da cirurgia, as pacientes foram avaliadas subjetivamente quanto ao grau de perda em relação ao que tinham antes. Assim, as próprias pacientes consideravam-se curadas, com melhora e inalteradas em relação à perda de urina, o que foi considerado como avaliação subjetiva. A existência de urgência ou urge-incontinência não caracterizou falha da cirurgia. Objetivamente, as pacientes foram avaliadas com ultrasonografia de colo vesical, teste do absorvente e estudo urodinâmico após pelo menos seis meses da cirurgia.

Depois de aproximadamente 46 meses para o TVT e de 52 meses para os casos de sling, as pacientes foram contatadas por telefone ou carta e entrevistadas quanto à avaliação subjetiva do tratamento. Responderam a esta avaliação $40(65,6 \%)$ pacientes do Grupo TVT e nove $(47,4 \%)$ do Grupo Sling.

O presente estudo foi previamente aprovado pelo Comitê de Ética em Pesquisa da Unifesp-EPM. Todas as pacientes foram informadas sobre a natureza do trabalho e aceitaram dele participar, assinando termo de consentimento pós-informado e carta de informação.

Para análise estatística, foi empregado o teste de Levene para avaliar a homogeneidade dos Grupos TVT e Sling em relação às variáveis: idade; IMC; número de gestações, de partos normais, fórcipe e cesárea, de abortos e tempo de perda urinária.

O teste do $X^{2}$ de Pearson foi empregado para análise da variável raça e para verificar a relação entre os grupos e as complicações (retenção urinária, infecção urinária, urgeincontinência e dificuldade para urinar) e para a avaliação da pressão abdominal no momento da perda.

Para avaliar as diferenças existentes entre os grupos em relação às medidas "antes" e "depois" foi efetuada a análise de variância (ANOVA) com medidas repetidas, em relação às variáveis: mobilidade do colo vesical, fluxo urinário máximo, fluxo urinário médio, resíduo pósmiccional, capacidade vesical no primeiro desejo miccional e capacidade vesical máxima.

Em todos os testes, foi usado o nível de significância igual a $5 \%(\boldsymbol{a}=0,05)$, sendo estatisticamente significantes os testes que apresentaram nível descritivo menor que $5 \%(\mathrm{p}<0,05)$.

\section{Resultados}

\section{Avaliação objetiva}

A análise da mobilidade do colo vesical foi feita antes e após seis meses da realização de ambas as cirurgias. Antes do procedimento, a mobilidade média do colo vesical foi de 17,5 mm no Grupo TVT e de 17,9 mm no Sling ( $\mathrm{p}=0,533$ ). Após a correção cirúrgica, houve diminuição significativa da mobilidade nos dois grupos, caindo para $13 \mathrm{~mm}$ no Grupo TVT $(\mathrm{p}<0,001)$ e para $10,9 \mathrm{~mm}$ no Sling $(\mathrm{p}<0,001)$. A diminuição foi estatisticamente semelhante (Tabela 2).

Em relação ao teste do absorvente, no Grupo TVT, apenas duas $(3,3 \%)$ pacientes apresentavam teste positivo no pós-operatório, e a perda foi considerada inalterada em relação à de antes da cirurgia. No Grupo Sling, cinco $(26,3 \%)$ mantiveram teste positivo, sendo duas com melhora e três inalteradas em relação ao volume de perda. Os dois grupos estudados exibiram similar taxa de melhora significante entre o pré- e o pós-operatório, que foi de $62,8 \mathrm{~g}$ para $3,4 \mathrm{~g}$ no Grupo TVT $(\mathrm{p}<0,001)$ e de $60,8 \mathrm{~g}$ para $8,5 \mathrm{~g}$ no Sling $(\mathrm{p}<0,001)$, conforme a Tabela 2.

Os dados do estudo urodinâmico estão dispostos na Tabela 3. Houve diminuição significativa, após seis meses, dos fluxos urinários máximo e médio em ambos os grupos. O resíduo pós-miccional foi semelhante no pré- e no pósoperatório das duas técnicas cirúrgicas, mas houve aumento significante entre o pré- e o pós-cirurgia de TVT, oscilando de 9,4 para 39,1 mL, respectivamente (Tabela 2).

Em relação à capacidade vesical no primeiro desejo miccional e à capacidade vesical máxima, os dois grupos foram semelhantes tanto no pré- quanto no pós-cirúrgico e também não houve alterações nos dois tempos analisados (Tabela 3).

Durante a cistometria realizada no pós-operatório, houve quatro (7\%) pacientes com perda de urina no Grupo TVT e quatro (20\%) no Sling. No entanto, a análise estatística mostrou que os grupos se comportaram da mesma maneira (Tabela 2).

A cura objetiva foi observada pela cistometria, quando não havia perda ao esforço solicitado. Assim, 93,4\% das mulheres do Grupo TVT e 78,9\% daquelas do Sling apresentavam cura objetiva (Tabela 2).

Separando-se as pacientes com defeito esfincteriano intrínseco e analisando a taxa de cura objetiva, observou-se que, dos 16 casos de IUE tipo III operadas por TVT, apenas uma $(6,2 \%)$ considerava-se não curada 
(7,5\%). Uma (2,5\%) das pacientes, considerada melhor com seis meses de pós-operatório, estava insatisfeita. A paciente que apresentava bexiga hiperativa antes da cirurgia mantinha-se com o mesmo quadro.

Quanto à cirurgia de sling, nove mulheres foram reavaliadas, em média, 52 meses depois e pudemos identificar cinco $(55,6 \%)$ delas curadas: uma $(11,1 \%)$ com sintomas irritativos e dificuldade de esvaziamento vesical persistente, uma $(11,1 \%)$ com recidiva da IUE, uma $(11,1 \%)$ que não teve melhora desde o pós-operatório inicial e uma $(11,1 \%)$ que estava apenas melhor que antes de ser operada.

\section{Complicações}

Descrevem-se as complicações observadas nas cirurgias na Tabela 4. A complicação mais freqüente da cirurgia de TVT foi a infecção urinária, em dez casos $(16,4 \%)$, semelhante à taxa observada no outro grupo $(\mathrm{p}=0,37)$. Observou-se retenção urinária em $42,1 \%$ dos casos de sling e em 9,8\% de TVT - valor este significativamente inferior $(\mathrm{p}=0,007)$. Houve relatos de dificuldade na micção em $6,5 \%$ das pacientes com TVT e em $21,1 \%$ das com sling $(\mathrm{p}=0,14)$. A urgência miccional com incontinência ocorreu em $21,1 \%$ das mulheres com sling e em 3,3\% das com TVT ( $\mathrm{p}=0,065)$.

Quanto ao tempo de sondagem vesical no pós-operatório, no Grupo TVT, $19(31,1 \%)$ pacientes tiveram a sonda vesical utilizada apenas durante a cirurgia e $23(37,7 \%)$ ficaram com sonda por 24 horas, em decorrência da raquianestesia. Dessas, 21 haviam se submetido também a outra cirurgia, sendo 18 colporrafias posteriores e perineoplastias, uma curetagem uterina, uma perineotomia e uma uretrólise. Os seis $(9,8 \%)$ casos de retenção urinária ficaram com a sonda entre seis e

Tabela 4 - Número e porcentagem de casos com complicações pós-operatórias entre pacientes que se submeteram às cirurgias de TVT ou sling de aponeurose

\begin{tabular}{|c|c|c|c|c|c|}
\hline \multirow{2}{*}{ Complicações } & \multicolumn{2}{|c|}{ TVT } & \multicolumn{2}{|c|}{ Sling } & \multirow{2}{*}{$\mathbf{p}$} \\
\hline & N & $\%$ & N & $\%$ & \\
\hline Retenção urinária & 6 & 9,8 & 8 & 42,1 & $0,007^{\star}$ \\
\hline Infecção urinária & 10 & 16,4 & 5 & 26,3 & 0,37 \\
\hline Dificuldade para urinar & 4 & 6,5 & 4 & 21,1 & 0,14 \\
\hline Urge-incontinência & 2 & 3,3 & 4 & 21,1 & 0,065 \\
\hline Perfuração vesical & 8 & 13,1 & 0 & 0 & \\
\hline Dor no baixo ventre & 7 & 11,4 & 2 & 10,5 & 0,091 \\
\hline Sangramento excessivo & 2 & 3,3 & 2 & 10,5 & 0,333 \\
\hline Infecção de parede & 0 & 0 & 2 & 10,5 & \\
\hline $\begin{array}{l}\text { Exteriorização de fio/ } \\
\text { faixa na vagina }\end{array}$ & 3 & 4,9 & 0 & 0 & \\
\hline $\begin{array}{l}\text { Deiscência de mucosa } \\
\text { vaginal }\end{array}$ & 0 & 0 & 1 & 5,2 & \\
\hline Pielonefrite & 1 & 1,6 & 1 & 5,2 & 0,50 \\
\hline Septicemia & 0 & 0 & 1 & 5,2 & \\
\hline $\begin{array}{l}\text { Necessidade de remoção } \\
\text { do sling }\end{array}$ & 0 & 0 & 1 & 5,2 & \\
\hline Deiscência de pele & 0 & 0 & 1 & 5,2 & \\
\hline
\end{tabular}

*Diferença significante (sling > TVT).
37 dias, com média de 18,5 dias. Permaneceu por um dia com a sonda uma paciente que teve sangramento maior, necessitando de tampão vaginal. Uma paciente desenvolveu bexiga hiperativa, com perda urinária em grande volume, e preferiu ficar sondada por nove dias; quando a medicação anticolinérgica foi introduzida, o quadro melhorou. Os oito casos de perfuração vesical permaneceram sondados de quatro a dez dias, com média de 6,4 dias. Ficaram com sonda por três dias duas pacientes que desenvolveram hematoma vesical pela passagem da agulha, identificados por cistoscopia. Uma paciente continuou com sonda por dois dias, porque houve grande dificuldade na realização da cistoscopia intra-operatória, com dor uretral.

No Grupo Sling, $11(57,9 \%)$ pacientes permaneceram sete dias com a sonda, conforme rotina do Setor e, após, tiveram micções espontâneas. Porém, oito $(42,1 \%)$ casos necessitaram de drenagem vesical por 14 a 54 dias, com média 24,3 dias. O tempo médio de sondagem vesical foi maior no Grupo Sling $(p=0,007)$.

\section{Discussão}

A partir de novos conceitos a respeito da fisiopatologia da IUE, em particular a teoria integral proposta por Petros e Ulmsten $^{11}$, a importância do equilíbrio entre a musculatura e os ligamentos pélvicos - especialmente o pubouretral - fez surgir nova opção cirúrgica: a TVT. Com ela, deixa-se de lado a necessidade de repor o colo vesical em posição retropúbica; apenas se refaz, com faixa de prolene, a ação dos ligamentos pubouretrais, que funcionariam estabilizando e ocluindo a uretra durante aumentos de pressão abdominal. No entanto, a técnica clássica e padrão-ouro para a correção da afecção, a cirurgia de Burch, apresenta excelentes resultados ao reposicionar o colo em topografia intra-abdominal. Isso demonstra que a fisiopatologia da IUE não é única, havendo vários mecanismos envolvidos e ainda não totalmente desvendados.

Portanto, aceita-se que a correção da IUE deve levar em consideração, ao menos, três possibilidades: elevação do colo vesical, estabilização da uretra média ou compressão uretral, como nos casos de esfíncteres artificiais ${ }^{2}$. Desse modo, a escolha da técnica cirúrgica deve se basear em experiências comprovadas na literatura, com seguimento adequado e fácil reprodutibilidade. A técnica ideal é, obviamente, aquela com melhores resultados, menos complicações e menos invasiva.

Considerando-se como padrão-ouro a cirurgia de Burch, estudos randomizados e multicêntricos compararam seus resultados aos do TVT, concluindo serem as taxas de cura semelhantes, sendo o TVT, porém, procedimento mais rápido e com menor tempo de internação e recuperação $0^{3,18,19}$.

Mesmo quando são avaliadas pacientes com IUE por defeito esfincteriano intrínseco ou incontinência urinária mista, as taxas de cura são satisfatórias ${ }^{10,20}$. Em nossa série, 
as pacientes com pressão de perda inferior a $60 \mathrm{cmH}_{2} \mathrm{O}$ no pré-operatório ficaram curadas em $93,7 \%$ com TVT e em $70 \%$ com sling.

Com relação aos slings, Bai et al. ${ }^{19}$ encontraram melhores taxas de sucesso nos casos de sling pubovaginal do que nas cirurgias de TVT ou Burch, diferindo dos nossos resultados. No entanto, em seu estudo, o seguimento foi de apenas um ano.

Em um estudo, no qual se compararam pacientes operadas por sling de aponeurose, TVT ou sling sintético $\left(\right.$ Pelvicol $\left.^{\circledR}\right)$, por meio de questionários específicos para sintomas urinários, observou-se que aquelas operadas por sling de aponeurose ou TVT mostraram resultados semelhantes e superiores aos do Pelvicol ${ }^{\circledR 27}$. Ressalta-se, porém, que a metodologia desses autores foi bastante diferente da empregada no presente estudo, já que a avaliação foi feita por meio de questionários enviados e, portanto, de caráter subjetivo.

Mesmo quando se compara o TVT com o Burch laparoscópico, que tem incisões menores e melhor recuperação pós-operatória, o TVT apresenta vantagens em relação ao tempo de cirurgia e à morbidade ${ }^{21}$, embora as taxas de cura sejam similares ${ }^{22}$.

Em estudo prospectivo, randomizado, com 92 pacientes com IUE, Bai et al. ${ }^{19}$ encontraram, depois de 12 meses, taxas de cura maior nos casos operados com sling pubovaginal do que com TVT ou Burch.

Aparentemente, os resultados quanto à cura da IUE são bastante semelhantes quando se usam slings, Burch ou TVT. Portanto, é importante analisar a morbidade dessas técnicas. Em nosso estudo, identificamos índices de complicações semelhantes entre as pacientes operadas por TVT ou por sling. No entanto, aquelas com cirurgia de TVT relatavam, nas consultas pós-operatórias, alto grau de satisfação com o intra- e pós-operatório. Importante salientar que o fato de não ser necessária a sondagem vesical prolongada nos casos de TVT fez com que essa diferença de satisfação fosse evidente.

A retenção urinária é complicação freqüente nas cirurgias de correção da IUE. Entretanto, a comparação com dados de literatura é extremamente prejudicada devido à ampla variação do que é considerado como retenção. Assim, alguns autores só consideram retenção urinária se houver necessidade de autocateterização intermitente prolongada e outros descrevem como retenção urinária momentânea ou transitória, quando ocorre por poucos dias ${ }^{20,28,29}$. Em nossa série de TVT, apenas uma paciente permaneceu sondada por mais de um mês. Esse caso, em particular, apresentou retenção urinária aguda no segundo dia de pós-operatório, porém demorou a ser tratada; assim, quando se passou a sonda vesical, notou-se ampla distensão do detrusor, com saída de cerca de um litro de urina. Tal fato pode ter contribuído para a disfunção miccional prolongada. Isso alerta quanto à necessidade de rigoroso acompanhamento e de orientação à paciente no pós-operatório, com o intuito de detectar precocemente a retenção urinária.

Casos de hematomas descritos são pouco freqüentes, porém, o relato de grande sangramento em mulher usuária de anticoagulante nos alerta sobre esse risco, levando-nos a inquirir sempre na anamnese pré-operatória sobre esse aspecto e evitando operar pacientes com incontinência urinária nessas condições.

Lesão vascular séria é rara; deve-se orientar bem a paciente que se submeterá à cirurgia com anestesia local sobre os riscos de movimentos intempestivos durante o procedimento. Além disso, devemos nos certificar de que a anestesia e a sedação estejam adequadas, para que não haja riscos desnecessários. Em nossa série, a maioria absoluta das pacientes foi operada com anestesia local e não tivemos nenhum problema quanto a isso.

Nas cirurgias de sling, a retirada da faixa de aponeurose poderia concorrer para aparecimento de hérnias, o que não foi observado nos nossos casos. Quanto à extrusão da faixa na vagina, identificamos o problema em $4,9 \%$ dos casos; todos tiveram a mucosa vaginal ressuturada sobre a faixa, evoluindo com sucesso. Notamos que, em todos esses casos, a faixa seccionou a mucosa vaginal durante sua inserção, provavelmente por ter sido passada muito superficialmente. Falconer et al. ${ }^{30}$ estudaram a reação histológica das faixas de prolene e encontraram mínima reação infamatória, justificando, com isso, a ausência de rejeição da faixa em mais de 80.000 procedimentos realizados.

Como pudemos observar em nossos resultados, tanto as pacientes operadas por TVT quanto por sling obtiveram bons resultados quanto à perda de urina. No entanto, aquelas com TVT permaneceram menos tempo sondadas e referiram maior taxa de cura subjetiva do que as com sling. A cura objetiva, no entanto, foi similar. Isso decorre, provavelmente, da satisfação quanto ao procedimento em si, do menor tempo necessário para recuperação, da menor taxa de retenção urinária e da melhora da urgência miccional.

Notamos também, nas entrevistas no pós-operatório, que as pacientes com TVT ficaram muito satisfeitas com o tamanho das incisões e com a rapidez de sua recuperação para suas atividades habituais.

No entanto, é importante salientar que, nos serviços que não dispõem de recursos financeiros para adquirir faixas sintéticas, as cirurgias de Burch (para hipermobilidade) e de sling (para as recidivas, defeito esfincteriano, mulheres obesas ou hipermobilidade) também podem ser realizadas com segurança quanto aos resultados e complicações.

Assim, concordamos com os autores que já afirmaram que a técnica de TVT pode ser considerada como padrão-ouro para correção de IUE, já que tem resultados equivalentes aos da cirurgia de Burch, sendo menos invasiva. Técnicas alternativas de acesso, como a via transobturadora, vêm 
sendo atualmente utilizadas, para evitar o espaço retropúbico, visando diminuir o risco de perfuração vesical, uretral, vascular ou de alças intestinais ${ }^{31}$. Com isso, maior número de pacientes poderão se beneficiar com modalidades cirúrgicas cada vez menos invasivas e com resultados altamente satisfatórios.

\section{Referências}

1. International Continence Society. Committee on Standardisation of Terminology. The standardization of terminology of lower urinary tract function. In: Ostergard DR, Bent AE, editors. Ostergard's urogynecology and pelvic floor dysfunction. 5th ed. Baltimore: Williams \& Wilkins; 2003. p. 391-402.

2. Stanton SL. Some reflections on tension-free vaginal tape: a new surgical procedure for treatment of female urinary incontinence. Int Urogynecol J. 2001;12 Suppl 2:S1-S2. ok

3. Albo ME, Richter HE, Brubaker L, Norton P, Kraus SR, Zimmern PE, et al. Burch colposuspension versus fascial sling to reduce urinary stress incontinence. N Engl J Med. 2007;356(21):2143-55.

4. Wall LL, Norton PA. Surgical management of stress urinary incontinence. In: Wall LL, Norton PA, editors. Practical urogynecology. Baltimore: Williams \& Wilkins; 1993. p. 153-90.

5. Horback NS. Suburethral sling procedures. In: Ostergard DR, Bent $A E$, editors. Urogynecology and urodynamics: theory and practice. 4th ed. Baltimore: Williams \& Wilkins; 1996. p. 569-79.

6. American Urogynecologic Society. Indications for a suburethral fascial sling. Washington, DC:AUGS. 1996. p. 1-5.

7. Haab F, Trockman BA, Zimmern PE, Leach GE. Results of pubovaginal sling for the treatment of intrinsic sphincteric deficiency determined by questionnaire analysis. J Urol. 1997;158(5):1738-41.

8. Chaikin DC, Rosenthal J, Blaivas JG. Pubovaginal fascial sling for all types of stress urinary incontinence: long-term analysis. J Urol. 1998; 160(4): 1312-6.

9. Leach GE, Dmochowski RR, Appell RA, Blaivas JG, Hadley HR, Luber $K M$, et al. Female Stress Urinary Incontinence Clinical Guidelines Panel summary report on surgical management of female stress urinary incontinence. The American Urological Association. J Urol. 1997; 158(3 Pt 1):875-80.

10. Silva-Filho AL, Triginelli SA, Noviello MB, Santos-Filho AS, Pires CR, Cunha-Melo JR. Pubovaginal sling in the treatment of stress urinary incontinence for urethral hypermobility and intrinsic sphincteric deficiency. Int Braz J Urol. 2003;29(6):540-4.

11. Petros PE, Ulmsten U. An integral theory and its method for the diagnosis and management of female urinary incontinence. Scand J Urol Nephrol Suppl. 1993;153:1-93.

12. Ulmsten $U$, Henriksson L, Johnson P, Varhos $G$. An ambulatory surgical procedure under local anesthesia for treatment of female urinary incontinence. Int Urogynecol J Pelvic Floor Dysfunct. $1996 ; 7(2): 81-5$.

13. Briones Mardones $G$, Jiménez Cidre $M$, Fernández Fernández E, Perales Cabanas L, Pozo Mengual B, Sanz Migueláñez JL, et al. Treatment of female stress urinary incontinence with the TVT (tension-free vaginal tape) system: our experience. Actas Urol Esp. $2001 ; 25(6): 423-9$.

14. Mutone N, Mastropietro M, Brizendine E, Hale D. Effect of tensionfree vaginal tape procedure on urodynamic continence indices. Obstet Gynecol. 2001 ;98(4):638-45.

15. Meschia M, Pifarotti P, Bernasconi F, Guercio E, Maffiolini M, Magatti F, Spreafico L. Tension-Free vaginal tape: analysis of outcomes and complications in 404 stress incontinent women. Int Urogynecol J Pelvic Floor Dysfunct. 2001;12 Suppl 2:S24-27.
16. Rezapour M, Ulmsten U. Tension-Free vaginal tape (TVT) in women with recurrent stress urinary incontinence-a long term follow up. Int Urogynecol J Pelvic Floor Dysfunct. 2001;12 Suppl 2:S9-11.

17. Drouin J, Tessier J, Bertrand PE, Schick E. Burch colposuspension: long-term results and review of published reports. Urology. 1999;54(5):808-14.

18. Liapis $A$, Bakas $P$, Creatsas $G$. Burch colposuspension and tensionfree vaginal tape in the management of stress urinary incontinence in women. Eur Urol. 2002;41(4):469-73.

19. Bai SW, Sohn WH, Chung DJ, Park JH, Kim SK. Comparison of the efficacy of Burch colposuspension, pubovaginal sling and tension-free vaginal tape for stress urinary incontinence. Int J Gynaecol Obstet. 2005;91(3):246-51.

20. Debodinance $P$, Delporte $P$, Engrand JB, Boulogne $M$. Tension-free vaginal tape (TVT) in treatment of urinary stress incontinence: 3 years experience involving 256 operations. Eur J Obstet Gynecol Reprod Biol. 2002;105(1):49-58.

21. Ustün Y, Engin-Ustün Y, Güngör M, Tezcan S. Tension-free vaginal tape compared with laparoscopic Burch urethropexy. J Am Assoc Gynecol Laparosc. 2003;10(3):386-9.

22. Paraiso MF, Walters MD, Karram MM, Barber MD. Laparoscopic Burch colposuspension versus tension-free vaginal tape: a randomized trial. Obstet Gynecol. 2004;104(6):1249-58.

23. Rezapour M, Ulmsten U. Tension-Free vaginal tape (TVT) in women with mixed stress urinary incontinence - a long-term follow-up. Int Urogynecol J Pelvic Floor Dysfunct. 2001;12 Suppl 2:S15-18.

24. Ryhammer AM, Djurhuus JC, Laurberg S. Pad testing in incontinent women: a review. Int Urogynecol J Pelvic Floor Dysfunct. 1999; 10(2): $111-5$.

25. Petros PP. The intravaginal slingplasty operation, a minimally invasive technique for cure of urinary incontinence in the female. Aust N Z J Obstet Gynaecol. 1996;36(4):453-61.

26. McGuire EJ, Lytton B. Pubovaginal sling procedure for stress incontinence. J Urol. 1978;119(1):82-4.

27. Liapis A, Bakas $P$, Lazaris D, Creatsas $G$. Tension-free vaginal tape in the management of recurrent stress urinary incontinence. Arch Gynecol Obstet. 2004;269(3):205-7.

28. Abouassaly R, Steinberg JR, Lemieux M, Marois C, Gilchrist LI, Bourque JL, et al. Complications of tension-free vaginal tape surgery: a multi-institutional review. BJU Int. 2004;94(1):110-3.

29. Morgan DM, Dunn RL, Fenner DE, Faerber G, DeLancey JO, McGuire EJ, et al. Comparative analysis of urinary incontinence severity after autologous fascia pubovaginal sling, pubovaginal sling and tension-free vaginal tape. J Urol. 2007;177(2):604-8.

30. Falconer C, Söderberg M, Blomgren, Ulmsten U. Influence of different sling materials on connective tissue metabolism in stress urinary incontinent women. Int Urogynecol J Pelvic Floor Dysfunct. 2001;12 Suppl 2:S19-23

31. Lee KS, Han DH, Choi YS, Yum SH, Song SH, Doo CK, et al. A prospective trial comparing tension-free vaginal tape and transobturator vaginal tape inside-out for the surgical treatment of female stress urinary incontinence: 1-year follow up. J Urol. 2007;177(1):214-8. 